Int. J. Electrochem. Sci., 12 (2017) 4990 - 4999

\title{
On-chip Hematocrit Correction for Whole Blood Glucose Amperometric Sensing Strip Using a Post-Measurement Potential Step
}

\author{
Chih-Wei Weng, Tzong-Jih Cheng, Richie L. C. Chen and Bo-Chuan Hsieh*
}

Biosensor Lab, Department of Bio-Industrial Mechatronics Engineering, National Taiwan University, No. 1, Sec. 4, Roosevelt Rd., Taipei 10617, Taiwan.

*E-mail: hsiehpc@ntu.edu.tw

doi: $10.20964 / 2017.06 .87$

Received: 27 February 2017 / Accepted: 19 April 2017 / Published: 12 May 2017

The accuracy of a point-of-care whole blood glucose sensor can be considerably influenced by hematocrit; therefore, an on-chip Hct correction protocol was developed and incorporated to meet the clinical demands. An impulse of DC $0.3 \mathrm{~V}$ was first applied for detecting blood glucose amperometrically, followed by an additional 3.2 V DC impulse for Hct estimation. Without Hct correction, the results of blood glucose tests were negatively correlated with Hct levels, and the glucose values were overestimated by $38.8 \%$ and underestimated by $43.8 \%$ when the Hct levels were $9 \%$ and $70 \%$, respectively. On the other hand, all the mean biases of blood glucose tests with 5 designed Hct levels $(9 \%, 25 \%, 43 \%, 55 \%$ and $70 \%)$ were less than $\pm 10 \%$ by utilizing the proposed Hct correction method. The method was insensitive to several representative interfering chemicals.

Keywords: Hematocrit compensation, post-measurement potential step, blood glucose sensor, strip

\section{$\underline{\text { FULL TEXT }}$}

(C) 2017 The Authors. Published by ESG (www.electrochemsci.org). This article is an open access article distributed under the terms and conditions of the Creative Commons Attribution license (http://creativecommons.org/licenses/by/4.0/). 